\title{
Phase diagram and thermodynamics of the Polyakov linear sigma model with three quark flavors
}

\author{
Hong $\mathrm{Mao}^{1,2,3}$, Jinshuang $\mathrm{Jin}^{2}$, and Mei Huang ${ }^{1,3}$ 胡 \\ 1. Institute of High Energy Physics, Chinese Academy of Sciences, Beijing 100049, China \\ 2. Department of Physics, Hangzhou Normal University, Hangzhou 310036, China \\ 3. Theoretical Physics Center for Science Facilities, \\ Chinese Academy of Sciences, Beijing 100049, China
}

\begin{abstract}
The phase diagram at finite temperature and density is investigated in the framework of the Polyakov linear sigma model (PLSM) with three light quark flavors in the mean field approximation. It is found that in the PLSM, the three phase transitions, i.e, the chiral restoration of $u, d$ quarks, the chiral restoration of $s$ quark, and the deconfinement phase transition are independent. There exists two-flavor quarkyonic phase at low density and three-flavor quarkyonic phase at high density. The critical end point (CEP) which separating the crossover from the first-order line in the PLSM model is located at $\left(T_{E}, \mu_{E}\right)=(188 \mathrm{MeV}, 139.5 \mathrm{MeV})$. In the transition region the thermodynamic properties and bulk viscosity over entropy density ratio $\zeta / s$ are also discussed in the PLSM.
\end{abstract}

PACS numbers: 12.38.Aw, 12.38.Mh, 12.39.Fe, 14.65.Bt

\section{INTRODUCTION}

It is widely believed that at sufficiently high temperatures and densities there is a quantum chromodynamics (QCD) phase transition between normal nuclear matter and quark-gluon plasma (QGP), where quarks and gluons are no longer confined in hadrons [1]. The theoretical and experimental investigation of QGP is one of the most challenging problems in high energy physics. The main target of heavy ion experiments at the Relativistic Heavy Ion collider (RHIC), the forthcoming Large Hadron Collider (LHC) and FAIR at GSI is to create such form of matter and study its properties.

There are two different phase transition which are associated with two opposite quark mass limits. For $N_{f}$ massless quark flavors, the QCD Lagrangian possesses a chiral $U\left(N_{f}\right)_{R} \times U\left(N_{f}\right)_{L}=S U\left(N_{f}\right)_{R} \times S U\left(N_{f}\right)_{L} \times U(1)_{V} \times U(1)_{A}$ symmetry, here $V=R+L$, while $A=R-L$. However this symmetry does not appear in the low energy particle spectrum, it is spontaneously broken to the diagonal $S U\left(N_{f}\right)_{V}$ group of vector transformation by a non-vanishing expectation value of the quark-antiquark condensate, $\left\langle\bar{q}_{R} q_{L}\right\rangle \neq 0$. This process involves $N_{f}^{2}$ Goldstone bosons which dominate the low-energy dynamics of the theory. The $U(1)_{V}$ symmetry is always respected and thus plays no role in the symmetry breaking pattern considered in the following discussion. The axial $U(1)_{A}$ symmetry is broken to $Z\left(N_{f}\right)_{A}$ by instanton effects [2] 3]. Consequently, one of the $N_{f}^{2}$ Goldstone bosons becomes massive, leaving $N_{f}^{2}-1$ Goldstone bosons. The $S U\left(N_{f}\right)_{R} \times S U\left(N_{f}\right)_{L} \times U(1)_{A}$ group is also explicitly broken by the effects of nonzero quark masses. The $N_{f}^{2}-1$ low energy degrees of freedom then become pseudo-Goldstone bosons. For $M \leq N_{f}$ degenerate

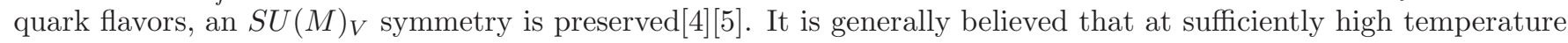
and density there should be a transition from ordinary hadronic matter to a chirally symmetric plasma of quark and gluons. At temperature of about $170 \mathrm{MeV}$, Lattice QCD calculations indicate that this symmetry is restored[6]. The order of the phase transition seems to depend on the mass of the non-strange $u$ and $d$ quarks, and the mass of the strange quark $m_{s}$, and at the temperature on the order of $200 \mathrm{MeV}$, heavier quark flavors do not play an essential role.

On the contrary, in the heavy quark limit, QCD reduces to a pure $S U\left(N_{c}\right)$ gauge theory which is invariant under a global $Z\left(N_{c}\right)$ center symmetry, where the Polyakov loop serves as an order parameter [0, 8, 9, 10]. In general, the Polyakov loop is a complex scalar field and it is related to the free energy of a static quark in the gluon medium, it vanishes in the confining phase means that the quark has infinite free energy and takes a finite value in the deconfined phase. It is linked to the $Z\left(N_{c}\right)$ center symmetry of the $S U\left(N_{c}\right)$ gauge theory, thus the confining phase is center symmetric, while the center symmetry is spontaneously broken in the deconfined phase. In the present of dynamical

\footnotetext{
*Electronic address: maohong@hznu.edu.cn

${ }^{\dagger}$ Electronic address: huangm@mail.ihep.ac.cn
} 
quarks the center symmetry is explicitly broken. No order parameter is established for the deconfinement transition in this case, but the Polyakov loop still serves as an indicator of a rapid crossover towards deconfinement 11. On the other hand, the chiral transition has a well-defined order parameter in the chiral limit of massless quarks: the quark-antiquark condensate, $\left\langle\bar{q}_{R} q_{L}\right\rangle \neq 0$, which and its dynamical generation, is the basic element of the linear sigma model and the Nambu-Jona-Lasinio model.

Recently, the effective chiral models for QCD with $U\left(N_{f}\right)_{R} \times U\left(N_{f}\right)_{L}$ symmetry, such as the Nambu-JonaLasinio model(NJL) or, via bosonization, the linear sigma model (LSM) could be combined with the Polyakov loop which allows to investigate both, the chiral and the deconfinement phase transition. These models named as the Polyakov-Nambu-Jona-Lasinio model (the PNJL) 12, 13, 14, and the Polyakov linear sigma model (PLSM) or the Polyakov quark meson model $(\mathrm{PQM})$ 15, 16, 17, are widely studied in recent years. In the PNJL and PLSM model, where quarks develop quasiparticle masses by propagating in the chiral condensate, while they couple at the same time to a homogeneous background (temporal) gauge field representing the Polyakov loop dynamics. The full QCD thermodynamics at zero and finite quark chemical potential have been investigated in Refs. 15, 16] 18, 19, 20, 21, 22, 23, 24, 25, 26, 27, 28, 29, 30]. However, most studies focus on the PNJL model with two and three quark flavors. As we know both of the NJL model and the LSM model for the phenomenology of QCD can be parameterized to describe equally well the vacuum structure at $T=\mu=0 \mathrm{MeV}$, but these models treat the contribution of the Dirac sea differently, in the NJL model it is included explicitly up to a momentum cutoff $\Lambda$, while in the sigma model this contribution is renormalized out. So that the extended PLSM model which is augmented with the Polyakov loop has the benefit of renormalizability. In this paper, calculations for the thermodynamic potential and the phase diagram will be performed at the mean-field level as that of the PNJL model, however, eventually, such model will be investigated beyond mean-field by the loop expansion 31, especially by using the CJT effective potential[32].

In present paper, we extend the Polyakov linear sigma model with two quark flavors [15, 16] to three quark flavor 17 to investigate the phase diagram at finite chemical potential. Moreover, since the study of the equation of state in QCD with the $2+1$ quark flavors by the lattice QCD simulations with almost physical quark masses have recently been reported in Ref[33 at zero chemical potential but finite temperature, it is interesting to describe QCD thermodynamics in the PLSM with three quark flavors, and compare with the recent results of the lattice simulations at zero chemical potential, especially, the essentials of QCD thermodynamics around the critical temperature $T_{c}$. The outline of the paper is as follows: in the next section we introduce the PLSM model with three quark flavors, some symmetry breaking patterns in the vacuum are briefly discussed, and parameters are fixed. In Sect. III, after obtaining the effective potential in the mean field approximation, we explore the phase diagram at $T-\mu$ plane. Section IV is devoted to derive the thermodynamic properties of the system at zero chemical potential, i.e. the pressure, the equation of state, the square of the speed of sound, the specific heat, the trace anomaly of the energy-momentum tensor and the bulk viscosity, all these thermodynamical observables are compared with the lattice QCD data. At the end, we give discussions and summary in Sec.V.

\section{THE MODEL}

Following Ref. [15], we introduce a generalized Lagrangian of the linear sigma model for $N_{f}=3$ quarks and $N_{c}=3$ color degrees with quarks coupled to a spatially constant temporal background gauge field representing Polyakov loop dynamics (the Polyakov-linear-sigma model or the PLSM in short), the Lagrangian reads [17]

$$
\mathcal{L}=\mathcal{L}_{\text {chiral }}-\mathcal{U}\left(\phi, \phi^{*}, T\right)
$$

where we have separated the contribution of chiral degrees of freedom and the Polyakov loop.

The chiral part of the Lagrangian, $\mathcal{L}_{\text {chiral }}=\mathcal{L}_{q}+\mathcal{L}_{m}$ [] [34], of the $S U(3)_{R} \times S U(3)_{L}$ symmetric linear sigma model with three quark flavors consists of the fermionic part

$$
\mathcal{L}_{q}=\sum_{f} \bar{\psi}_{f}\left(i \gamma^{\mu} D_{\mu}-g T_{a}\left(\sigma_{a}+i \gamma_{5} \pi_{a}\right)\right) \psi_{f}
$$

and the purely mesonic contribution

$$
\begin{aligned}
\mathcal{L}_{m}= & \operatorname{Tr}\left(\partial_{\mu} \Phi^{\dagger} \partial^{\mu} \Phi-m^{2} \Phi^{\dagger} \Phi\right)-\lambda_{1}\left[\operatorname{Tr}\left(\Phi^{\dagger} \Phi\right)\right]^{2} \\
& -\lambda_{2} \operatorname{Tr}\left(\Phi^{\dagger} \Phi\right)^{2}+c\left[\operatorname{Det}(\Phi)+\operatorname{Det}\left(\Phi^{\dagger}\right)\right] \\
& +\operatorname{Tr}\left[H\left(\Phi+\Phi^{\dagger}\right)\right],
\end{aligned}
$$

the sum is over the three flavors ( $\mathrm{f}=1,2,3$ for $\mathrm{u}, \mathrm{d}, \mathrm{s})$. In the above equation we have introduced a flavor-blind Yukawa coupling $g$ of the quarks to the mesons and the coupling of the quarks to a background gauge field $A_{\mu}=\delta_{\mu 0} A_{0}$ via 
the covariant derivative $D_{\mu}=\partial_{\mu}-i A_{\mu}$. The $\Phi$ is a complex $3 \times 3$ matrix and is defined in terms of the scalar $\sigma_{a}$ and pseudoscalar $\pi_{a}$ meson nonets,

$$
\Phi=T_{a}\left(\sigma_{a}+i \pi_{a}\right)
$$

The $3 \times 3$ matrix $H$ breaks the symmetry explicitly and is chosen as

$$
H=T_{a} h_{a},
$$

where $h_{a}$ are nine external fields. The $T_{a}=\lambda_{a} / 2$ are the generators of the $U(3)$ symmetry, $\lambda_{a}$ are the Gell-Mann matrices with $\lambda_{0}=\sqrt{\frac{2}{3}} \mathbf{1}$. The $T_{a}$ are normalized to $\operatorname{Tr}\left(T_{a} T_{b}\right)=\delta_{a b} / 2$ and obey the $U(3)$ algebra with $\left[T_{a}, T_{b}\right]=i f_{a b c} T_{c}$ and $\left\{T_{a}, T_{b}\right\}=d_{a b c} T_{c}$ respectively, here $f_{a b c}$ and $d_{a b c}$ for $a, b, c=1, \ldots, 8$ are the standard antisymmetric and symmetric structure constants of $S U(3)$ group and

$$
f_{a b 0} \equiv 0, \quad d_{a b 0}=\sqrt{\frac{2}{3}} \delta_{a b} .
$$

In Eq.(3), $m^{2}$ is the tree-level mass square of the fields in the absence of symmetry breaking, $\lambda_{1}$ and $\lambda_{2}$ are two possible quartic coupling constants, and $c$ is the cubic coupling constant. The terms in the first line of Eq.(3) are invariant under $U(3)_{R} \times U(3)_{L} \cong U(3)_{V} \times U(3)_{A}$ transformations, the determinant terms are invariant under $S U(3)_{R} \times S U(3)_{L} \cong S U(3)_{V} \times S U(3)_{A}$, but break the $U(1)_{A}$ symmetry explicitly, these terms arise from the $U(1)_{A}$ anomaly of the QCD vacuum. The last term in Eq.(3) breaks the axial and the $S U(3)_{V}$ vector symmetries explicitly. A non-vanishing vacuum expectation value for $\Phi,\langle\Phi\rangle \equiv T_{a} \bar{\sigma}_{a}$, breaks the chiral symmetry spontaneously. Because the parity is not broken in the vacuum, there are no non-vanishing vacuum expectation values for fields $\pi_{a}$. The patterns of explicit symmetry breaking have been described in detail in Ref. [5] for the $U(3)_{V} \times U(3)_{A}$ model, in this work we will constrain our study on the case of explicit chiral symmetry breaking with $U(1)_{A}$ anomaly. Since $\langle\Phi\rangle$ must carry the quantum numbers of the vacuum, only the fields $\bar{\sigma}_{a}$ corresponding to the diagonal generators of $U(3)$ can be nonzero. The same holds for $h_{a}$, then the diagonal components $h_{0}, h_{3}$ and $h_{8}$ of the explicit symmetry breaking term could be nonzero. Furthermore due to the fact that the masses of the up and down quarks are approximately equal, $m_{u} \simeq m_{d}$, and the strange quark mass $m_{s}$ is larger than $m_{u}$ and $m_{d}$, in the following discussion, we restrict our study to $h_{0} \neq 0, h_{3}=0$ and $h_{8} \neq 0$. In this case the $S U(3)_{V} \times S U(3)_{A}$ symmetry is explicitly broken to $S U(2)_{V}$ isospin symmetry, and the $U(1)_{A}$ subgroup of the $U(3)_{A}$ symmetry is already explicitly broken by the instanton. Traditionally, the linear sigma model does not have quark degrees of freedom, the nonzero quark masses correspond to a term of the form Eq.(3) in the lagrangian, where the matrix $H$ is proportional to the quark mass matrix, the fields $H$ can be determined from the vacuum values for the pion and kaon masses, as well as the pion and kaon decay constants, the values for the constants of proportionality could be fixed at bare quark masses for $u, d$ and $s$ quarks, for example $m_{q}=10 \mathrm{MeV}, m_{s}=150 \mathrm{MeV}$ [35]. This means that the parameters in the purely mesonic Lagrangian carries the information of the bare quark mass, in order to avoid the double counting, such model do not introduce bare quark masses in Lagrangian (2).

The quantity $\mathcal{U}\left(\phi, \phi^{*}, T\right)$ is the Polyakov-loop effective potential expressed by the dynamics of the traced Polyakov loop

$$
\phi=\left(\operatorname{Tr}_{c} L\right) / N_{c}, \quad \phi^{*}=\left(\operatorname{Tr}_{c} L^{\dagger}\right) / N_{c}
$$

The Polyakov loop $L$ is a matrix in color space and explicitly given by

$$
L(\vec{x})=\mathcal{P} \exp \left[i \int_{0}^{\beta} d \tau A_{4}(\vec{x}, \tau)\right],
$$

with $\beta=1 / T$ being the inverse of temperature and $A_{4}=i A^{0}$. In the so-called Polyakov gauge, the Polyakov-loop matrix can be given as a diagonal representation [12]. The coupling between Polyakov loop and quarks is uniquely determined by the covariant derivative $D_{\mu}$ in the PLSM Lagrangian in Eq.(11), and in the chiral limit, this Lagrangian is invariant under the chiral flavor group, just like the original QCD Lagrangian. The trace of the Polyakov-loop, $\phi$ and its conjugate $\phi^{*}$ can be treated as classical field variables in this work.

The temperature dependent effective potential $\mathcal{U}\left(\phi, \phi^{*}, T\right)$ is used to reproduced the thermodynamical behavior of the Polyakov loop for the pure gauge case in accordance with lattice QCD data, and it has the $Z(3)$ center symmetry like the pure gauge QCD Lagrangian. In the absence of quarks, we have $\phi=\phi^{*}$ and the Polyakov loop is taken as an order parameter for deconfinement. For low temperatures, $\mathcal{U}$ has a single minimum at $\phi=0$, while at high temperatures it develops a second one which turns into the absolute minimum above a critical temperature $T_{0}$, and 
the $Z(3)$ center symmetry is spontaneously broken. In this paper, we will use the potential $\mathcal{U}\left(\phi, \phi^{*}, T\right)$ proposed in Ref. 13], which has a polynomial expansion in $\phi$ and $\phi^{*}$ :

$$
\frac{\mathcal{U}\left(\phi, \phi^{*}, T\right)}{T^{4}}=-\frac{b_{2}(T)}{2}|\phi|^{2}-\frac{b_{3}}{6}\left(\phi^{3}+\phi^{* 3}\right)+\frac{b_{4}}{4}\left(|\phi|^{2}\right)^{2},
$$

with

$$
b_{2}(T)=a_{0}+a_{1}\left(\frac{T_{0}}{T}\right)+a_{2}\left(\frac{T_{0}}{T}\right)^{2}+a_{3}\left(\frac{T_{0}}{T}\right)^{3} .
$$

A precision fit of the constants $a_{i}, b_{i}$ is performed to reproduce the lattice data for pure gauge theory thermodynamics and the behavior of the Polyakov loop as a function of temperature. The corresponding parameters are

$$
\begin{array}{r}
a_{0}=6.75, \quad a_{1}=-1.95, \quad a_{2}=2.625 \\
a_{3}=-7.44, \quad b_{3}=0.75, \quad b_{4}=7.5
\end{array}
$$

The critical temperature $T_{0}$ for deconfinement in the pure gauge sector is fixed at $270 \mathrm{MeV}$, in agreement with the lattice results.

The chiral part of the model involves seven parameter $m^{2}, \lambda_{1}, \lambda_{2}, c, g, h_{0}, h_{8}$ and two unknown condensates $\bar{\sigma}_{0}$ and $\bar{\sigma}_{8}$, which we take from [34], where they were fixed to reproduce some physical quantities in the hadronic sector. The vacuum condensates $\bar{\sigma}_{0}$ and $\bar{\sigma}_{8}$ are members of the scalar $\left(J^{p}=0^{+}\right)$nonet and both contain the up-down quark condensate and the strange quark condensate. It is more convenient to convert the condensates into a pure up-down quark condensate and strange condensate, this is achieved by an orthogonal basis transformation from the original octet-singlet basis $\left(\bar{\sigma}_{0}, \bar{\sigma}_{8}\right)$ to the up-down quark condensate $\sigma_{x}$ and the strange quark condensate $\sigma_{y}$ basis (for simplicity, we ignore the bar over the up-down quark condensate $\sigma_{x}$ and the strange quark condensate $\sigma_{x}$ ),

$$
\begin{aligned}
\sigma_{x} & =\sqrt{\frac{2}{3}} \bar{\sigma}_{0}+\frac{1}{\sqrt{3}} \bar{\sigma}_{8}, \\
\sigma_{y} & =\frac{1}{\sqrt{3}} \bar{\sigma}_{0}-\sqrt{\frac{2}{3}} \bar{\sigma}_{8} .
\end{aligned}
$$

As a result, when chiral symmetry breaks spontaneously, the fields $\langle\Phi\rangle$ acquires a non-vanishing vacuum expectation value, the constituent quark masses for $u, d$ and $s$ are defined to be

$$
\begin{array}{lrrr}
m_{q}=g \sigma_{x} / 2 & \text { for } & u, d, \\
m_{s}=g \sigma_{y} / \sqrt{2} & \text { for } & s,
\end{array}
$$

and the light quark sector decouples from the strange quark sector. At zero temperature and zero chemical potential, the parameters of the Lagrangian are fixed in a way that these masses agree with the observed value of pion mass $m_{\pi}=138 \mathrm{MeV}$ and the most commonly accepted value for sigma mass $m_{\sigma}=600 \mathrm{MeV}$. The values of the condensates are determined from the pion and kaon decay constants by means of the partially conserved axial-vector current relation (PCAS),

$$
\sigma_{x}=f_{\pi}, \quad \sigma_{y}=\frac{1}{\sqrt{2}}\left(2 f_{K}-f_{\pi}\right),
$$

and the decay constants, $f_{\pi}=92.4 \mathrm{MeV}$ and $f_{K}=113 \mathrm{MeV}$. The Yukawa coupling is usually fixed by requirement that the constituent quark mass in the vacuum, for $u$ and $d$ quarks, is about $1 / 3$ of the nucleon mass, which gives $g \simeq 6.5$. The strange constituent quark mass is predicted to be $m_{s} \simeq 433 \mathrm{MeV}$. The six parameters $m^{2}, \lambda_{1}, \lambda_{2}, c$, $h_{x}$ and $h_{y}$ are set for $m_{\sigma}=600 \mathrm{MeV}$ with the axial $U(1)_{A}$ anomaly: $m^{2}=(342.52 \mathrm{MeV})^{2}, \lambda_{1}=1.40, \lambda_{2}=46.48$, $c=4807.84 \mathrm{MeV}, h_{x}=(120.73 \mathrm{MeV})^{3}$ and $h_{y}=(336.41 \mathrm{MeV})^{3}$ 34].

\section{MEAN FIELD APPROXIMATION}

The standard approach for dealing with the thermodynamics of variable particles is via the grand canonical ensemble. Let us consider a spatially uniform system in thermodynamical equilibrium at temperature $T$ and quark chemical 
potential $\mu_{f}(f=u, d, s)$. In general, the grand partition function reads

$$
\begin{aligned}
\mathcal{Z} & =\operatorname{Tr} \exp \left[-\left(\hat{\mathcal{H}}-\sum_{f=u, d, s} \mu_{f} \hat{\mathcal{N}}_{f}\right) / T\right] \\
& =\int \prod_{a} \mathcal{D} \sigma_{a} \mathcal{D} \pi_{a} \int \mathcal{D} \psi \mathcal{D} \bar{\psi} \exp \left[\int_{x}\left(\mathcal{L}+\sum_{f=u, d, s} \mu_{f} \bar{\psi}_{f} \gamma^{0} \psi_{f}\right)\right],
\end{aligned}
$$

where $\int_{x} \equiv i \int_{0}^{1 / T} d t \int_{V} d^{3} x$ and $V$ is the volume of the system. The $u$ and $d$ quark chemical potentials is

$$
\mu_{u}=\frac{\mu_{B}}{3}+\frac{\mu_{I}}{2}+\frac{1}{3} \mu_{Y}, \quad \mu_{d}=\frac{\mu_{B}}{3}-\frac{\mu_{I}}{2}+\frac{1}{3} \mu_{Y}, \quad \mu_{s}=\frac{\mu_{B}}{3}-\frac{2}{3} \mu_{Y}
$$

where $\mu_{B}, \mu_{I}$ and $\mu_{Y}$ are the baryon, isospin and hypercharge chemical potentials respectively. In general, the three quark chemical potentials are independent, but in the following discussion, we assume the $S U(2)_{V}$ isospin symmetry by neglecting the slight mass difference between an $u$ - and $d$ - quark. Then the light quark chemical potentials become equal, $\mu_{q} \equiv \mu_{u}=\mu_{d}$, and there is no effect of the isospin density since the isospin chemical potential $\mu_{I}$ is zero. Also for simplicity and confronting our results with the other models, such as LSM and PNJL models with three quark flavors, we will only consider symmetric quark matter and define a uniform chemical potential $\mu \equiv \mu_{q}=\mu_{s}$ for $\mu_{Y}=0$.

We evaluate the partition function in the mean-field approximation similar to [34 36]. Thus we replace the meson fields by their expectation values in the action. In other words, we neglect both quantum and thermal fluctuations of the meson fields. The quarks and antiquarks are retained as quantum fields. The integration over the fermions yields a determinant which can be calculated by standard methods 37. This generates an effective potential for the mesons. Finally, we obtain the thermodynamical potential density as

$$
\Omega\left(T, \mu_{f}\right)=\frac{-T \ln \mathcal{Z}}{V}=U\left(\sigma_{x}, \sigma_{y}\right)+\mathcal{U}\left(\phi, \phi^{*}, T\right)+\Omega_{\bar{\psi} \psi},
$$

with the quarks and antiquarks contribution

$$
\begin{aligned}
\Omega_{\bar{\psi} \psi}= & -2 T N_{q} \int \frac{d^{3} \vec{p}}{(2 \pi)^{3}}\left\{\ln \left[1+3\left(\phi+\phi^{*} e^{-\left(E_{q}-\mu\right) / T}\right) \times e^{-\left(E_{q}-\mu\right) / T}+e^{-3\left(E_{q}-\mu\right) / T}\right]\right. \\
& \left.+\ln \left[1+3\left(\phi^{*}+\phi e^{-\left(E_{q}+\mu\right) / T}\right) \times e^{-\left(E_{q}+\mu\right) / T}+e^{-3\left(E_{q}+\mu\right) / T}\right]\right\} \\
& -2 T N_{s} \int \frac{d^{3} \vec{p}}{(2 \pi)^{3}}\left\{\ln \left[1+3\left(\phi+\phi^{*} e^{-\left(E_{s}-\mu\right) / T}\right) \times e^{-\left(E_{s}-\mu\right) / T}+e^{-3\left(E_{s}-\mu\right) / T}\right]\right. \\
& \left.+\ln \left[1+3\left(\phi^{*}+\phi e^{-\left(E_{s}+\mu\right) / T}\right) \times e^{-\left(E_{s}+\mu\right) / T}+e^{-3\left(E_{s}+\mu\right) / T}\right]\right\} .
\end{aligned}
$$

Here, $N_{q}=2, N_{s}=1$, and $E_{q}=\sqrt{\vec{p}^{2}+m_{q}^{2}}$ is the valence quark and antiquark energy for $u$ and $d$ quarks, for strange quark $s$, it is $E_{s}=\sqrt{\vec{p}^{2}+m_{s}^{2}}$, and $m_{q}, m_{s}$ is the constituent quark mass for $u, d$ and $s$ in Eqs.(13)(14). The purely mesonic potential is

$$
U\left(\sigma_{x}, \sigma_{y}\right)=\frac{m^{2}}{2}\left(\sigma_{x}^{2}+\sigma_{y}^{2}\right)-h_{x} \sigma_{x}-h_{y} \sigma_{y}-\frac{c}{2 \sqrt{2}} \sigma_{x}^{2} \sigma_{y}+\frac{\lambda_{1}}{2} \sigma_{x}^{2} \sigma_{y}^{2}+\frac{1}{8}\left(2 \lambda_{1}+\lambda_{2}\right) \sigma_{x}^{4}+\frac{1}{4}\left(\lambda_{1}+\lambda_{2}\right) \sigma_{y}^{4} .
$$

Minimizing the thermodynamical potential in Eq.(18) with respective to $\sigma_{x}, \sigma_{y}, \phi$ and $\phi^{*}$, we obtain a set of equations of motion

$$
\frac{\partial \Omega}{\partial \sigma_{x}}=0, \quad \frac{\partial \Omega}{\partial \sigma_{y}}=0, \quad \frac{\partial \Omega}{\partial \phi}=0, \quad \frac{\partial \Omega}{\partial \phi^{*}}=0 .
$$

The set of equations can be solved for the fields as functions of temperature $T$ and chemical potential $\mu$, and the solutions of these coupled equations determine the behavior of the chiral order parameter $\sigma_{x}, \sigma_{y}$ and the Polyakov loop expectation values $\phi, \phi^{*}$ as a function of $T$ and $\mu$.

\section{IV. $T-\mu$ PHASE DIAGRAM}

We now explore the phase diagram of the Polyakov linear sigma model at finite temperature and density. There are three different critical temperatures, i.e, $T_{c}^{q}$ for the chiral phase transition of $u, d$ quarks, $T_{c}^{s}$ for the chiral phase 
transition of $s$ quark, and $T_{c}^{d}$ for the deconfinement phase transition. In order to locate the critical temperatures, we define the temperature derivatives of the condensates: $\sigma_{x}^{\prime}=\partial \sigma_{x} / \partial T, \sigma_{y}^{\prime}=\partial \sigma_{y} / \partial T, \phi^{\prime}=\partial \phi / \partial T$ and $\phi^{* \prime}=\partial \phi^{*} / \partial T$. In the following, we will show the double peak structure of the temperature derivatives of the condensates and explain explicitly how we determine the critical temperatures.

\section{A. Phase transitions at zero density}

We firstly investigate the phase transitions at zero density. In Fig.1](a), the temperature dependence of the chiral condensates $\sigma_{x}$ for $u, d$ quarks, the chiral condensate $\sigma_{y}$ for $s$ quark and the Polyakov loop expectation value $\phi, \phi^{*}$ at $\mu=0 \mathrm{MeV}$ is shown in relative units. Here and in the following discussion, the chiral condensates are normalized by their zero-temperature value: $\sigma_{x 0}=92.4 \mathrm{MeV}$ and $\sigma_{y 0}=94.5 \mathrm{MeV}$ for $u, d$ and $s$ quarks, respectively. The temperature derivatives of the condensates $\sigma_{x}^{\prime}, \sigma_{y}^{\prime}, \phi^{\prime}$ and $\phi^{* \prime}$ are shown in Fig.1(b).

The temperature behavior of the chiral condensates and Polyakov loop condensate shows that the system experiences a crossover at zero chemical potential. The temperature derivative of the chiral condensate $\sigma_{x}$ for $u$ and $d$ quarks has only one peak at $T \simeq 200 \mathrm{MeV}$. The temperature derivative of the chiral condensates $\sigma_{y}$ for $s$ quark has two peaks, the left peak coincides with the peak of $\sigma_{x}^{\prime}$ at $T \simeq 200 \mathrm{MeV}$, the right peak shows up at $T \simeq 260 \mathrm{MeV}$. For vanishing chemical potential, we find $\phi=\phi^{*}$, and $\phi \simeq 1.1$ for $T \rightarrow \infty$. The temperature derivatives $\phi^{\prime}$ and $\phi^{* \prime}$ shows one peak and one pseudo-peak, and the peak coincides with the peak of $\sigma_{x}^{\prime}$ at $T \simeq 200 \mathrm{MeV}$.

Now we show how we determine the critical temperature of the crossover. When there is only one peak in the temperature derivative of the condensate, the location of the peak gives the critical temperature $T_{c}$. When there are two peaks in the temperature derivative of the condensate, for chiral phase transition, the critical temperature is determined by the peak temperature corresponding to $\sigma_{x, y}(T) / \sigma_{x, y}(T=0)<1 / 2$, and for deconfinement phase transition, the critical temperature is given by the peak or pseudo peak temperature corresponding to $\phi(T) / \phi(T \rightarrow$ $\infty)>1 / 2$. This definition of the critical temperature is different from that defined in Ref.[14.

The temperature derivatives of the condensates in Fig.1 (b) show that the deconfinement phase transition happens at a higher critical temperature $T_{c}^{d} \simeq 220 \mathrm{MeV}$, and the chiral restoration occurs at a smaller critical temperature $T_{c}^{q} \simeq 203.5 \mathrm{MeV}$. This result is similar to that obtained in the two-flavor Polyakov linear sigma model Ref. [16], and different from that in the three-flavor Nambu-Jona-Lasinio model with the Polyakov loop [14], where they find the simultaneous crossovers around $T_{c} \simeq 200 \mathrm{MeV}$. The critical temperature for the chiral restoration of the strange quark is at $T_{c}^{s} \simeq 260 \mathrm{MeV}$.

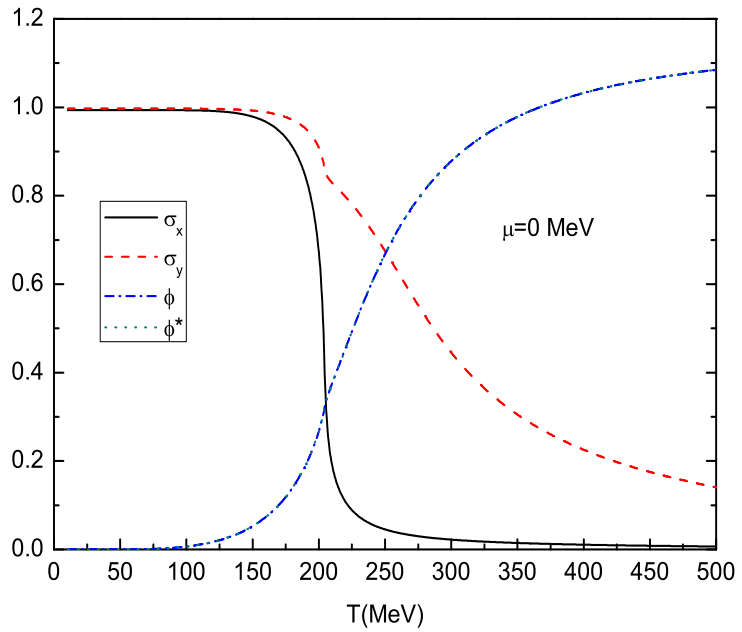

( a )

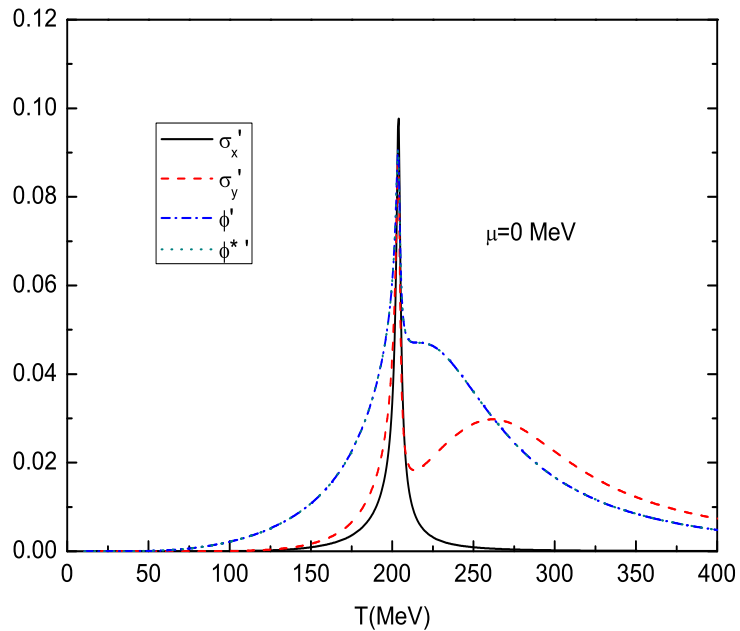

( b )

FIG. 1: (a) The normalized chiral condensate $\sigma_{x}, \sigma_{y}$ and the Polyakov loop $\phi, \phi^{*}$ as a function of temperature for $\mu=0$ $\mathrm{MeV}$. (b) Temperature derivatives of the normalized chiral condensate $\sigma_{x}, \sigma_{y}$ and the Polyakov loop $\phi, \phi^{*}$ as a function of temperature at $\mu=0 \mathrm{MeV}$. The Polyakov variable and $\sigma_{y}$ are scaled by a factor of 6 . 


\section{B. Phase transitions at finite density}

For fixing the chemical potential at $200 \mathrm{MeV}$, the order parameters and their temperature derivatives as functions of temperature are shown in Fig.2 (a) and (b), respectively.

It is found that the temperature derivative of the chiral condensate $\sigma_{x}$ for $u$ and $d$ quarks still has only one peak, at the peak temperature $T_{c}^{q} \simeq 173 \mathrm{MeV}$ of $\sigma_{x}^{\prime}$, the chiral condensate jumps from 0.8 to 0.15 , which indicates that the chiral phase transition for the $u$ and $d$ quarks is of first order phase transition.

The temperature derivative of chiral condensates $\sigma_{y}$ for $s$ quark has two peaks, the left peak coincides with the peak of $\sigma_{x}^{\prime}$ at $T_{c}^{q} \simeq 173 \mathrm{MeV}$, the right peak shows up at $T_{c}^{s} \simeq 240 \mathrm{MeV}$.

The values of the Polyakov loop $\phi$ and $\phi^{*}$ are different for nonvanishing chemical potential, due to the fact that the free energies of quark and antiquarks are different in the finite chemical potential [38, 39. When $\mu$ increases, the left peak moves down to a smaller temperature, which is always coincident with $T_{c}^{q}$, and the right pseudo-peak at zero chemical potential develops into a real peak and stays almost at the same temperature $T_{c}^{d} \simeq 220 \mathrm{MeV}$ as that for $\mu=0$.

Here are several remarks on the phase transitions. The left peak of $\sigma_{y}^{\prime}$ and $\phi^{\prime}\left(\phi^{* \prime}\right)$ always coincides with the peak of $\sigma_{x}^{\prime}$, which reflects the effective coupling among $\sigma_{x}, \sigma_{y}$ and $\phi\left(\phi^{*}\right)$. However, the coupling among the condensates does not necessarily induce the simultaneous phase transitions. From the above analysis, we can see that in general cases, the three phase transitions, i.e, the chiral restoration of $u, d$ quarks, the chiral restoration of $s$ quark, and the deconfinement phase transition are independent in the Polyakov linear sigma model. However the lattice result shows the simultaneous phase transitions of chiral restoration and deconfinement, it should be very interesting to investigate how to realize the simultaneous phase transitions in the Polyakov linear sigma model, and it deserves further efforts on understanding the correlation between the chiral phase transitions and deconfinement phase transition.

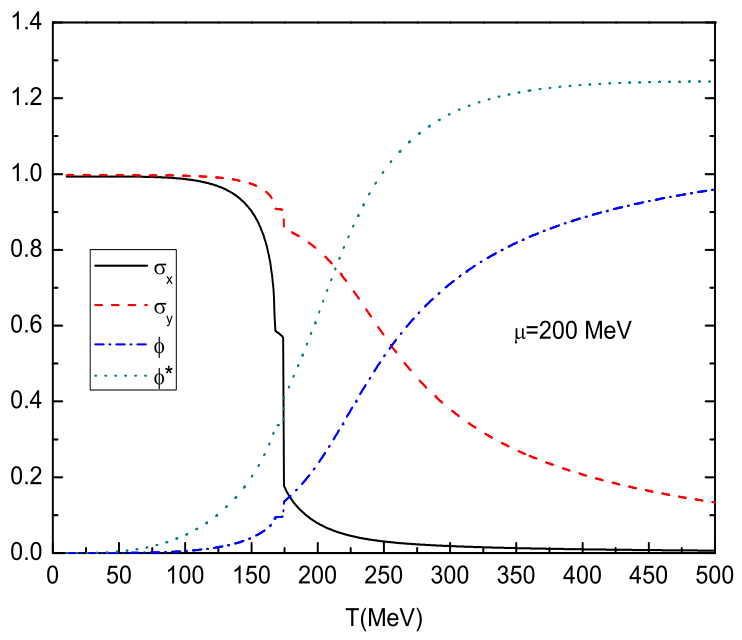

( a )

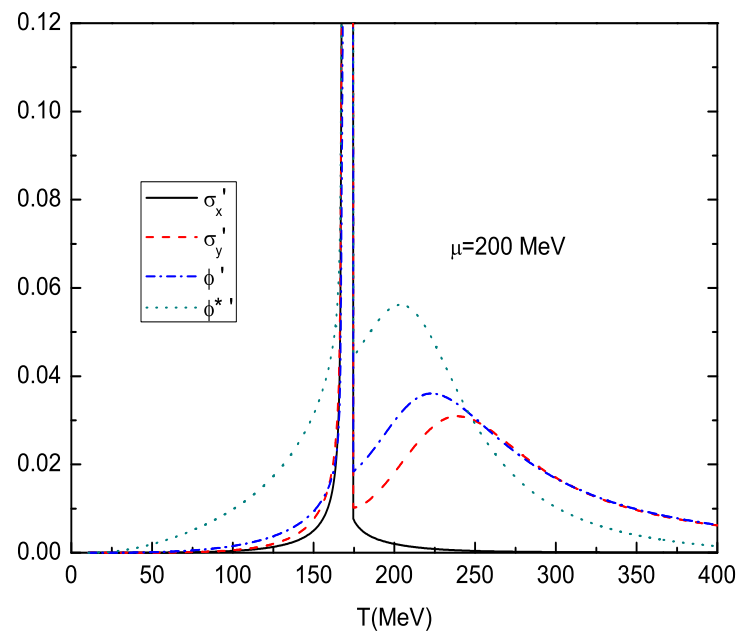

( b )

FIG. 2: (a) The normalized chiral condensate $\sigma_{x}, \sigma_{y}$ and the Polyakov loop $\phi, \phi^{*}$ as a function of temperature for $\mu=200$ MeV. (b) Temperature derivatives of the normalized chiral condensate $\sigma_{x}, \sigma_{y}$ and the Polyakov loop $\phi, \phi^{*}$ as a function of temperature at $\mu=200 \mathrm{MeV}$. The Polyakov variable and $\sigma_{y}$ are scaled by a factor of 6 .

\section{C. $T-\mu$ phase structure and the location of the critical end point}

We evaluate the chiral phase transitions of $u, d$ and $s$ quarks and deconfinement phase transition at finite temperature and finite density, and show the $T-\mu$ phase structure of the Polyakov linear sigma model in Fig.3 (a).

From Fig.1 and Fig.2, we see that the chiral phase transition for $s$ quark and the deconfinement phase transition for the Polyakov loop are always crossover, for two light flavors, there is a crossover in the low density region and a first-order phase transition in the high-density region, and in the middle exists a critical end point (CEP). In order to locate the critical end point, we define the quark number susceptibility $\chi_{q}=\partial^{2} \Omega / \partial \mu^{2}$, which should be divergent at the critical end point. In Fig. 3 (b), we plot the quark number susceptibility as a function of the temperature for different chemical potentials. For the Polyakov linear sigma model, the result shows that the critical end point is around $\left(T_{E}, \mu_{E}\right)=(188 \mathrm{MeV}, 139.5 \mathrm{MeV})$, which is close to the lattice result $\left(T_{E}, \mu_{E}\right)=\left(162 \pm 2 \mathrm{MeV}, \mu_{E}=\right.$ 
$120 \pm 13 \mathrm{MeV}$ ) 40. For the linear sigma model without the Polyakov loop, the critical end point is located at $\left(T_{E}, \mu_{E}\right) \simeq(92.5 \mathrm{MeV}, 216 \mathrm{MeV})$. The critical chemical potential $\mu_{E}$ in PLSM is much lower than that in the PNJL model with three quark flavors where the predicted critical end point is $\mu_{E}>300 \mathrm{MeV}[23,24]$.

The chiral phase transition for the strange quark and the deconfinement phase transition in the $T-\mu$ plane are shown in Fig.3 (a) by the dash-dotted line and dotted line, respectively. It is found that with the increase of chemical potential, the critical temperature for strange quark to restore chiral symmetry decreases. However, for the deconfinement phase transition, with the increase of chemical potential, the deconfinement critical temperature keeps almost a constant around $220 \mathrm{MeV}$. It can be seen that in the Polyakov linear sigma model, there exists twoflavor quarkyonic phase [41] at low density, where the $u, d$ quarks restore chiral symmetry but still in confinement, and three-flavor quarkyonic phase at high density, where the $u, d, s$ quarks restore chiral symmetry but still in confinement.

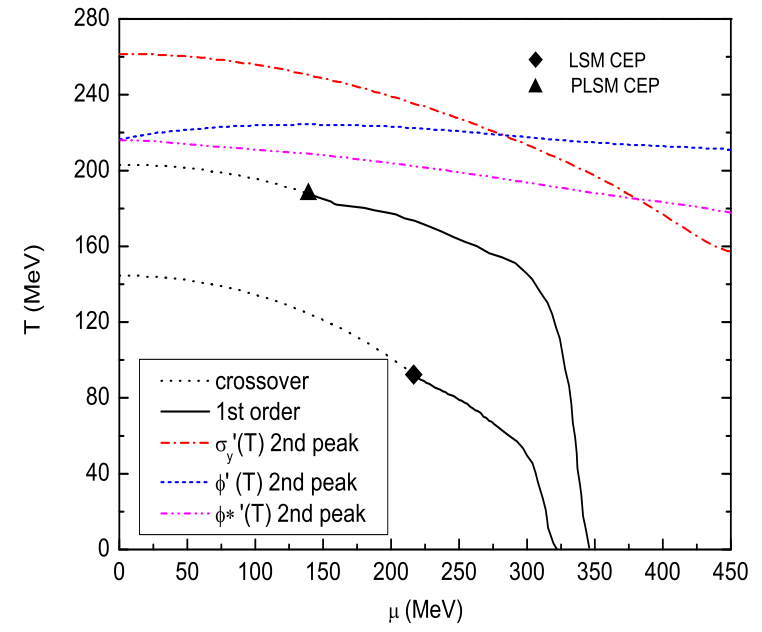

(a)

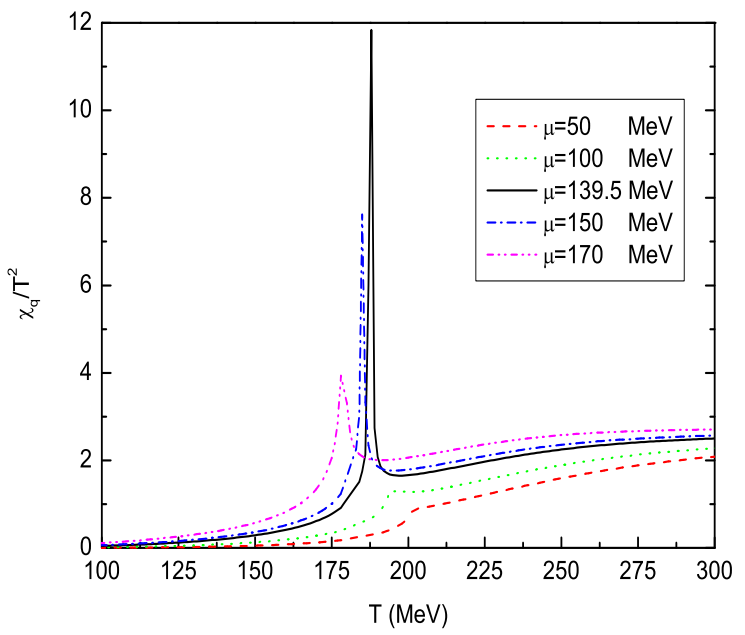

( b )

FIG. 3: (a) The $T-\mu$ phase diagram in the Polyakov linear sigma model. (b) the quark number susceptibility $\chi_{q}$ for different chemical potentials as function of $T$.

\section{THERMODYNAMIC PROPERTIES OF THE POLYAKOV LINEAR SIGMA MODEL}

In order to investigate the influence of the Polyakov loop on the equilibrium thermodynamics, we calculate several thermodynamic quantities. All information of the system is contained in the grand canonical potential which is given by $\Omega$ in Eq.(18), evaluated at the mean field level. The entropy density is determined by taking the derivative of effective potential with respect to the temperature, i.e,

$$
s=-\partial \Omega(\phi) / \partial T
$$

As the standard treatment in lattice calculation, we introduce the normalized pressure density $p$ which is normalized to vanish at $T=\mu=0$ and the energy density $\varepsilon$ as

$$
p=-\Omega, \varepsilon=-p+T s .
$$

The equation of state $p(\varepsilon)$ is an important input into hydrodynamics. The square of the speed of sound $C_{s}^{2}$ is related to $p / \varepsilon$ and has the form of

$$
C_{s}^{2}=\frac{\mathrm{d} p}{\mathrm{~d} \varepsilon}=\frac{s}{T \mathrm{~d} s / \mathrm{d} T}=\frac{s}{C_{v}},
$$

where

$$
C_{v}=\partial \varepsilon / \partial T
$$

is the specific heat. At the critical temperature, the entropy density as well as the energy density change most quickly with temperature, thus one expect that $C_{s}^{2}$ should have a minimum at $T_{c}$. The trace anomaly of the energy-momentum 
tensor $\mathcal{T}^{\mu \nu}$

$$
\Delta=\frac{\mathcal{T}^{\mu \mu}}{T^{4}} \equiv \frac{\varepsilon-3 p}{T^{4}}=T \frac{\partial}{\partial T}\left(p / T^{4}\right)
$$

is a dimensionless quantity, which is also called the "interaction measure".

In Fig. 4 8, we show the trace anomaly, energy density, pressure density, equation of state parameter, specific heat and sound velocity square as functions of the scaled temperature $T / T_{c}$.

The result of the trace anomaly of the energy-momentum tensor $\mathcal{T}^{\mu \nu}$ at zero density is shown in Fig.4(a) in comparison with lattice data for $N_{\tau}=6$ [33]. It is shown that the trace anomaly approaches the conformal value 0 at high temperature in both the linear sigma model and the Polyakov linear sigma model, which agrees with the lattice result. The trace anomaly shows a peak around $T_{c}$ in the linear sigma model, and the height is only half of that of lattice result. After including the Polyakov loop, the peak of the trace anomaly appears at a higher temperature $1.5 T_{c}$ comparing with the lattice result, where the peak shows up at around $1.1 T_{c}$. We will show later that the appearance of the peak in the trace anomaly is not related to the phase transition, but the change rate of the trace anomaly is related to the phase transition.

The result of the trace anomaly of the energy-momentum tensor at different chemical potentials as function of the scaled temperature $T / T_{c}$ is shown in Fig. (b). It is observed that the trace anomaly changes smoothly around $T_{c}$ in the case of zero chemical potential when the system experiences a crossover, but changes sharply in the case of fist order phase transition at high density. From Fig. 5 , we can see clearly that the behavior of the trace anomaly at $T_{c}$ resembles that of the energy density and the entropy density at $T_{c}$. We will show latter that the change rate of the energy density and/or entropy density at $T_{c}$ determine the critical behavior of other thermal quantities like the specific heat, the sound velocity square.

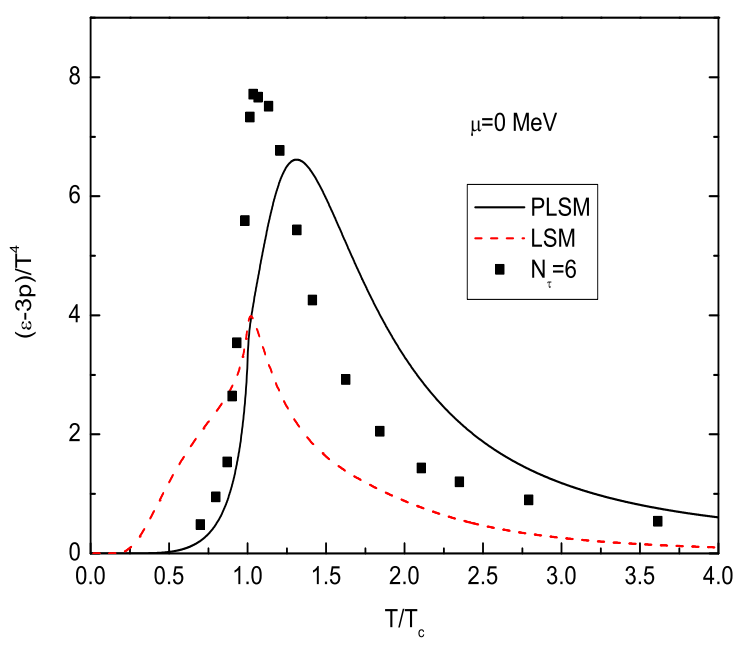

( a )

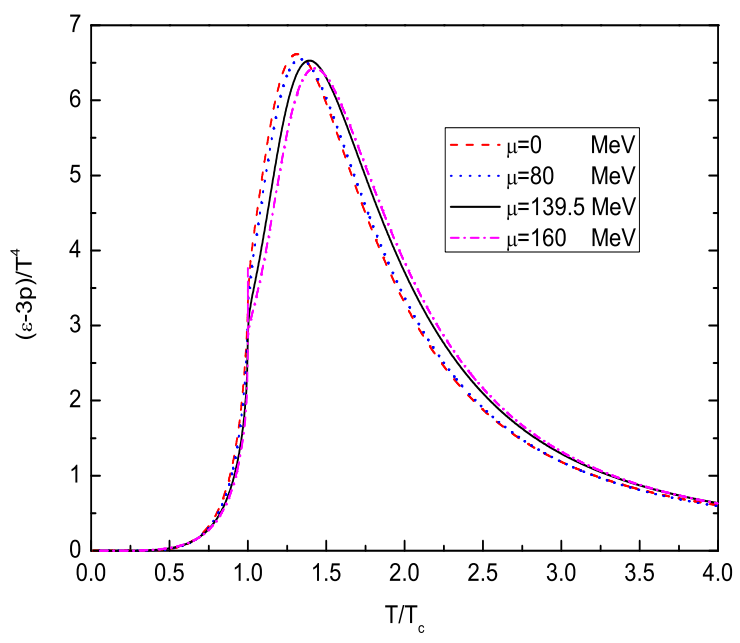

( b )

FIG. 4: (a) The scaled trace anomaly $(\varepsilon-3 p) / T^{4}$ for $\mu=0 \mathrm{MeV}$. The Polyakov linear sigma model prediction (solid line) and the linear sigma model prediction (dashed line) are compared with $N_{f}=2+1$ lattice QCD data for $N_{\tau}=6$. Lattice data taken from Ref. 33]. (b) The scaled trace anomaly $(\varepsilon-3 p) / T^{4}$ in the Polyakov linear sigma model for different chemical potentials as functions of $T / T_{c}$.

Fig.6 shows the pressure density over energy density $p / \varepsilon$, which is represented in terms of equation-of-state (EOS) parameter, at zero density and finite density, respectively. We observe that the pressure density over energy density increases with temperature and saturates at high temperature. Both the linear sigma model and the Polyakov linear sigma model give very similar results at high temperature, the pressure density over energy density $p / \varepsilon$ saturates at a value smaller than $1 / 3$. Another common feature of the $p / \varepsilon$ in the linear sigma model and the Polyakov linear sigma model is that there is a bump appearing at low temperature region, which is also observed in the lattice result. Around the critical temperature $T_{c}$, the pressure density over energy density $p / \varepsilon$ shows a downward cusp. However, the minimum value of the $p / \varepsilon$ around $T_{c}$ is 0.2 in the linear sigma model, which is much larger than the result from the Polyakov linear sigma model and the lattice QCD data. For the Polyakov linear sigma model, the minimum of $p / \varepsilon$ around $T_{c}$ is 0.075 , which is consistent with the lattice QCD data [33]. When the chemical potential increases, from Fig. (b), we can see that the minimum of the $p / \varepsilon$ around $T_{c}$ decreases.

We show the specific heat $C_{v}$ as a function of the scaled temperature $T / T_{c}$ in Fig. 月. It it shown that there is a sharp peak arising at $T_{c}$ both in the Polyakov linear sigma model and linear sigma model. At high chemical potential when 


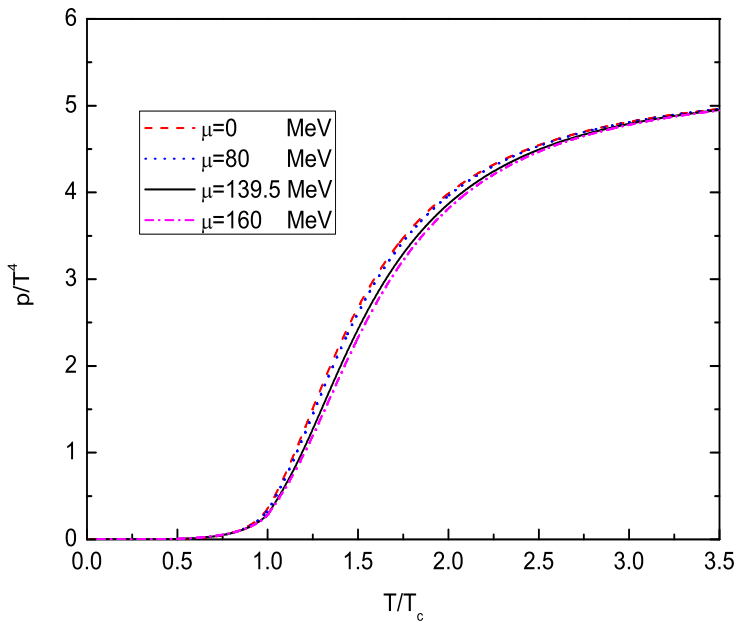

( a )

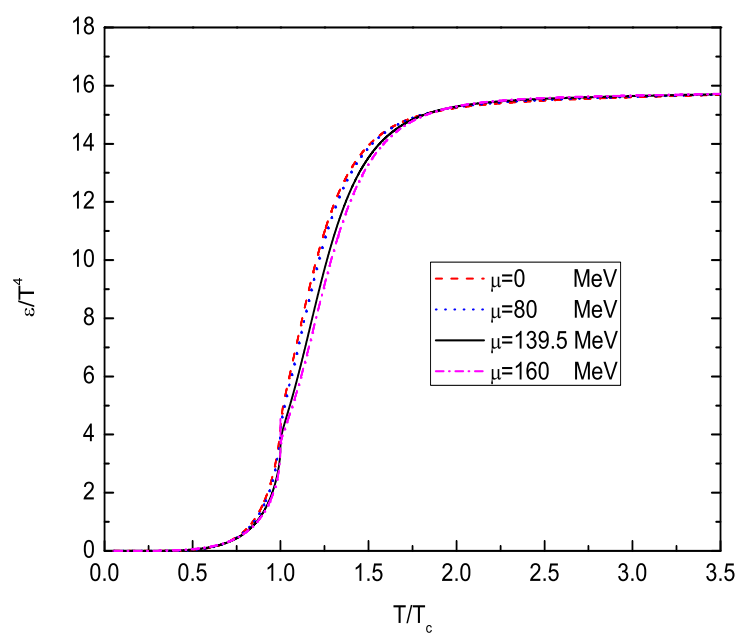

( b )

FIG. 5: (a) The scaled pressure density $p / T^{4}$ in the Polyakov linear sigma model for different chemical potentials as functions of $T / T_{c}$. (b) The scaled energy density $\varepsilon / T^{4}$ in the Polyakov linear sigma model for different chemical potentials as functions of $T / T_{c}$.

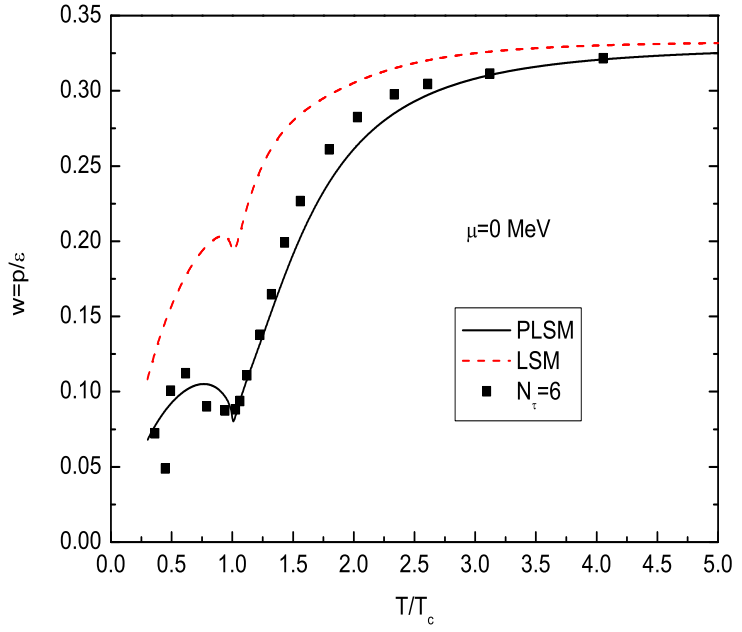

( a )

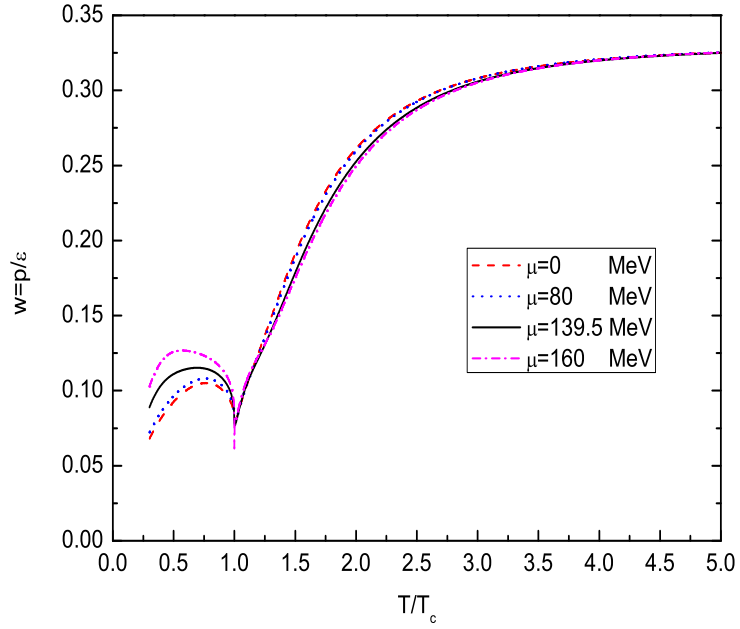

( b )

FIG. 6: (a) The equation-of-state parameter $w(T)=p(T) / \varepsilon(T)$ for $\mu=0 \mathrm{MeV}$. The Polyakov linear sigma model prediction (solid line) and the linear sigma model prediction (dash line) are compared with $N_{f}=2+1$ lattice QCD data for $N_{\tau}=6$. Lattice data taken from Ref. 33]. (b) The equation-of-state parameter $w(T)=p(T) / \varepsilon(T)$ in the PLSM for different chemical potentials as functions of $T / T_{c}$.

the phase transition is of first order, the specific heat diverges. From the definition of the specific heat $C_{v}=\partial \varepsilon / \partial T$, it is easy to understand that the appearance of the peak is due the fast change of the energy density at the critical temperature $T_{c}$. The other issue presented in the temperature behavior of $C_{v}$ in Fig.7 is that there is a small second peak at higher temperature, which is more visible in the Polyakov linear sigma model than that in the linear sigma model. This result is consistent with the PLSM model with two quark flavors 16 .

Fig.8 shows the sound velocity square $C_{s}^{2}$ as a function of the scaled temperature. In conformal field theories including free field theory, the sound velocity square is always $1 / 3$. In both the linear sigma model and the Polyakov linear sigma model, the $C_{s}^{2}$ saturates at a value smaller than $1 / 3$ at high temperature. However, near the critical temperature $T_{c}$, a downward cusp shows up. At zero density, the minimum of the sound velocity square $C_{s}^{2}$ at $T_{c}$ is around 0.05 and 0.16 in the Polyakov linear sigma model and the linear sigma model, respectively, and the value of $C_{s}^{2}$ at $T_{c}$ in the Polyakov linear sigma model is close the lattice result. When the chemical potential increases, the minimum of $C_{s}^{2}$ at $T_{c}$ decreases, and it approaches 0 in the case of first order phase transition. 


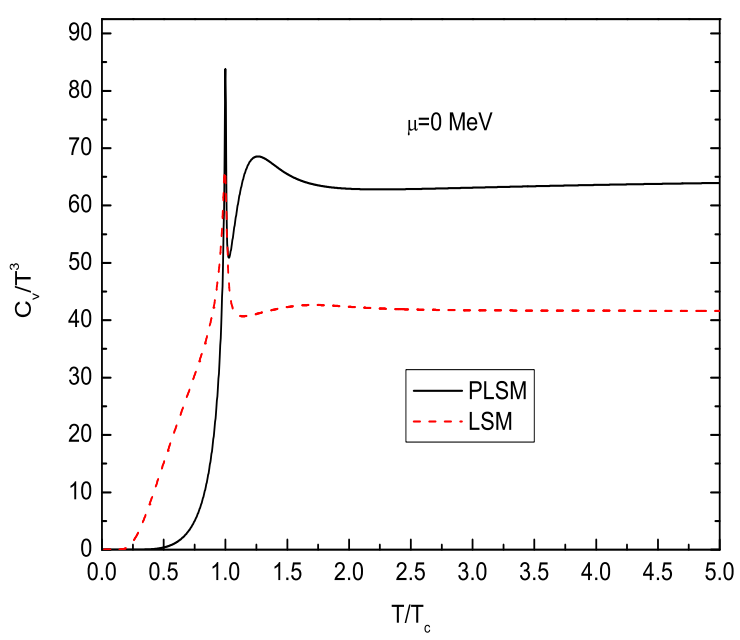

( a )

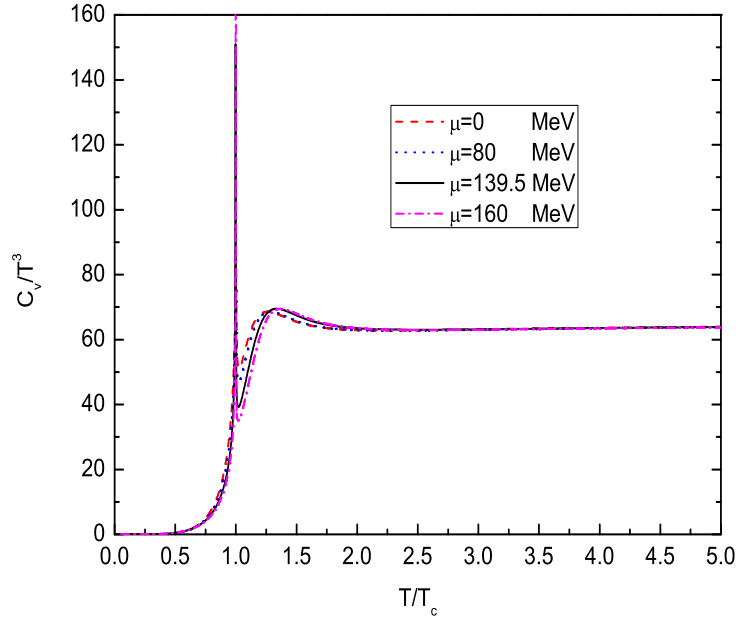

( b )

FIG. 7: (a) The specific heat $C_{v}$ as a function of the temperature for $\mu=0 \mathrm{MeV}$. The solid line denotes the Polyakov linear sigma model prediction and the dashed line denotes the linear sigma model prediction. (b)The specific heat $C_{v}$ in the PLSM for different chemical potentials as functions of $T / T_{c}$.

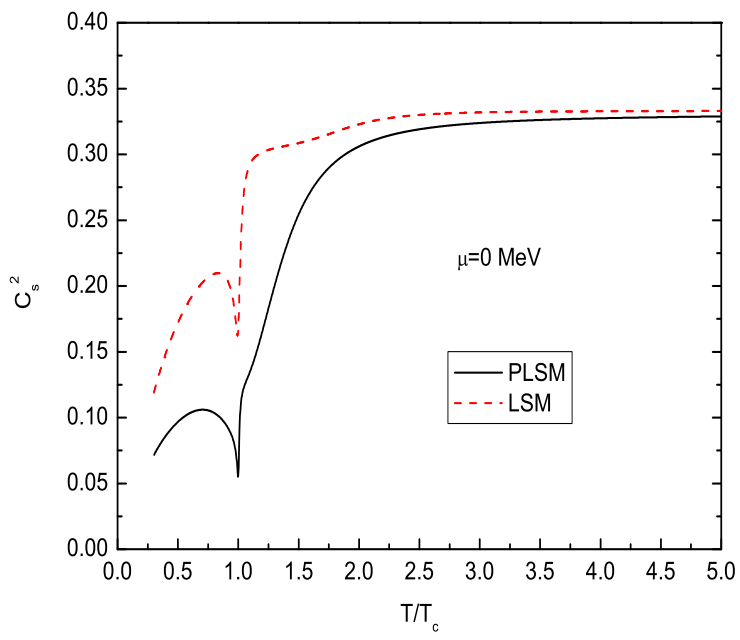

( a )

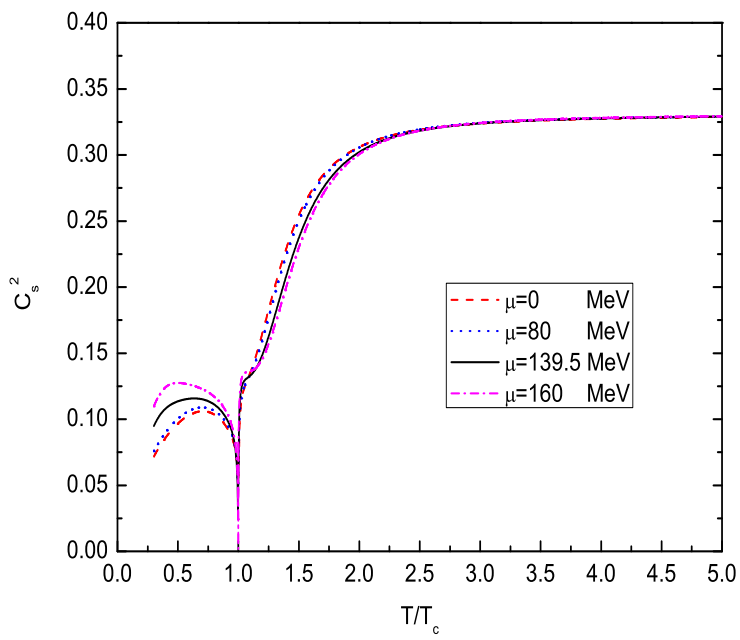

( b )

FIG. 8: (a) The sound velocity square $C_{s}^{2}$ as a function of the temperature for $\mu=0 \mathrm{MeV}$. The solid line denotes the Polyakov linear sigma model prediction and the dashed line denotes the linear sigma model prediction. (b) The sound velocity square $C_{s}^{2}$ in the PLSM for different chemical potentials as functions of $T / T_{c}$.

\section{THE BULK VISCOSITY OVER ENTROPY DENSITY RATIO IN THE POLYAKOV LINEAR SIGMA MODEL}

The bulk viscosity is related to the correlation function of the trace of the energy-momentum tensor $\theta_{\mu}^{\mu}$ :

$$
\zeta=\frac{1}{9} \lim _{\omega \rightarrow 0} \frac{1}{\omega} \int_{0}^{\infty} d t \int d^{3} r e^{i \omega t}\left\langle\left[\theta_{\mu}^{\mu}(x), \theta_{\mu}^{\mu}(0)\right]\right\rangle .
$$

According to the result derived from low energy theorem, in the low frequency region, the bulk viscosity takes the form of 42 43]

$$
\begin{aligned}
\zeta & =\frac{1}{9 \omega_{0}}\left\{T^{5} \frac{\partial}{\partial T} \frac{(\varepsilon-3 p)}{T^{4}}+16\left|\varepsilon_{v}\right|\right\}, \\
& =\frac{1}{9 \omega_{0}}\left\{-16 \varepsilon+9 T S+T C_{v}+16\left|\varepsilon_{v}\right|\right\} .
\end{aligned}
$$


with the negative vacuum energy density $\varepsilon_{v}=\Omega_{v}=\left.\Omega(\phi)\right|_{T=0}$, and the parameter $\omega_{0}=\omega_{0}(T)$ is a scale at which the perturbation theory becomes valid. From the above formula, we can see that the bulk viscosity is proportional to the specific heat $C_{v}$ near phase transition, thus $\zeta / s$ behaves as $1 / C_{s}^{2}$ near $T_{c}$ in this approximation.

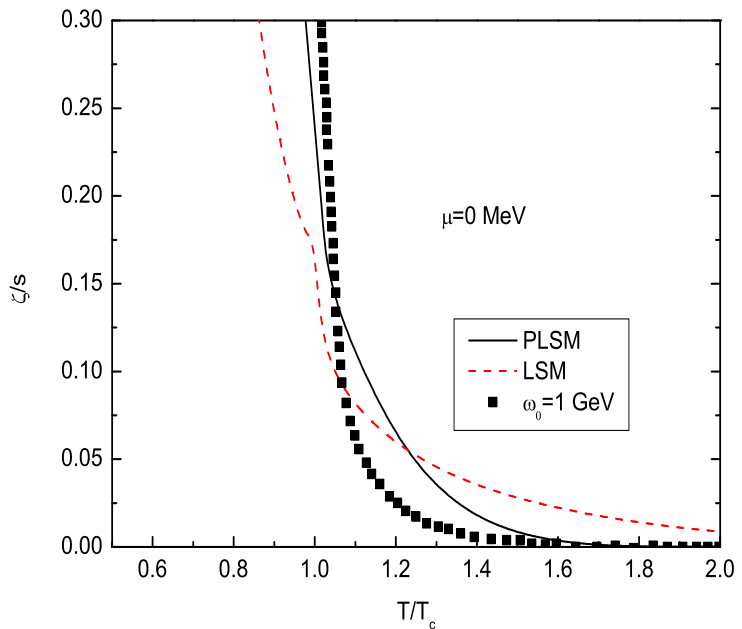

( a )

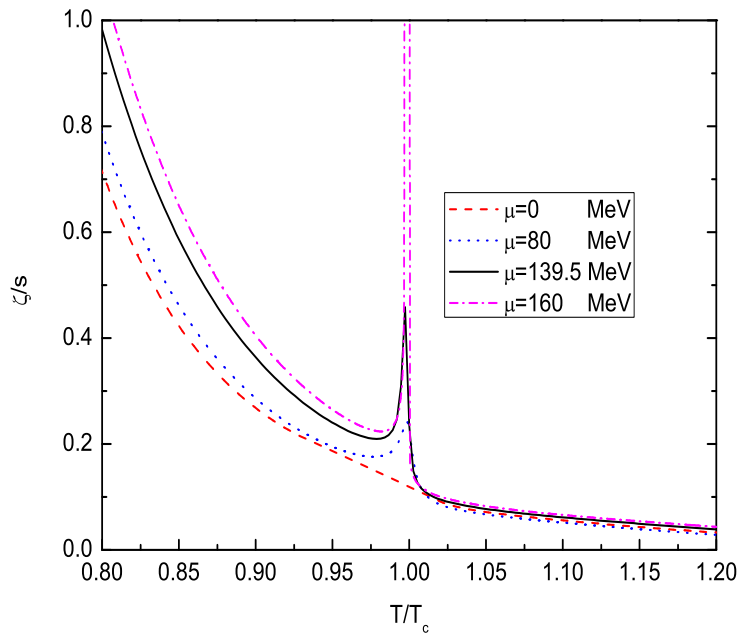

( b )

FIG. 9: (a) The bulk viscosity over entropy density ratio $\zeta / s$ as a function of the temperature for $\mu=0 \mathrm{MeV}$. The solid line denotes the Polyakov linear sigma model prediction and the dashed line denotes the linear sigma model prediction. Lattice data taken from Ref. 43]. (b) The bulk viscosity over entropy density ratio $\zeta / s$ in the PLSM for different chemical potentials as functions of $T / T_{c}$.

In Fig.9 (a) and (b), we plot the bulk viscosity over entropy density ratio $\zeta / s$ as a function of the temperature for zero chemical potential and finite chemical potential, respectively. From Fig.9 (a), it is shown that, at zero chemical potential $\mu=0$, the bulk viscosity over entropy density $\zeta / s$ decreases monotonically with the increase of the temperature in both the Polyakov linear sigma model and linear sigma model, and at high temperature, $\zeta / s$ reaches its conformal value 0 . In [43], the bulk viscosity over entropy density of the three flavor system is extracted from lattice result, which is shown in Fig.9 (a) by the square. It is observed that $\zeta / s$ in PLSM near phase transition is in very good agreement with the lattice result in [43], i.e, it rises sharply near phase transition. From Fig.9 (b), which shows $\zeta / s$ as function of the scaled temperature $T / T_{c}$ for different chemical potentials with $\mu=0,80,139.5,160$ $\mathrm{MeV}$, We can see that when the chemical potential increases up to $\mu=80 \mathrm{MeV}$, there is an upward cusp appearing in $\zeta / s$ right at the critical temperature $T_{c}$. With the increase of the chemical potential, the upward cusp becomes sharper, and the height of the cusp increases. At the critical end point $\mu_{E}$ and when $\mu>\mu_{E}$ for the first order phase transition, $\zeta / s$ becomes divergent at the critical temperature.

The critical behavior of $\zeta / s$ is determined by the shape of the trace anomaly or the energy density around the critical temperature $T_{c}$. In the case of first order phase transition, the energy density has a sudden change at the critical temperature, thus $\zeta / s$ diverges at $T_{c}$. In the case of crossover, $\zeta / s$ can exhibit different behaviors near phase transition: 1) When the energy density or entropy density changes slowly around $T_{c}, \zeta / s$ monotonically but slowly rises up with the decrease of temperature. 2) When the energy density or the entropy density changes quickly near $T_{c}, \zeta / s$ monotonically but quickly rises up when the temperature decreases. 3) When the energy density or entropy density changes very quickly near $T_{c}$, an upward cusp of $\zeta / s$ appears at $T_{c}$, i.e, $\zeta / s$ firstly rises quickly when the temperature decreases to $T_{c}$, then jumps fast and eventually rises up when the temperature drops further away from $T_{c}$. These behaviors have been analyzed in Ref. [44 for a toy model.

It is expected that one can distinguish whether the system experiences a first order phase transition or a crossover from observables which are sensitive to the bulk viscosity at RHIC experiments. As mentioned in Refs. 45, 46. that a sharp rise of bulk viscosity near phase transition induces an instability in the hydrodynamic flow of the plasma, and this mode will blow up and tear the system into droplets. It would be interesting to investigate in more detail how different behaviors of $\zeta / s$ affect the observables.

However, it is noticed that the results of bulk viscosity in this paper are based on Eq. (28), where the ansatz for the spectral function

$$
\frac{\rho(\omega, \overrightarrow{0})}{\omega}=\frac{9 \zeta}{\pi} \frac{\omega_{0}^{2}}{\pi\left(\omega^{2}+\omega^{2}\right)}
$$


has been used in the small frequency, and $\omega_{0}$ is a scale at which the perturbation theory becomes valid. In our calculation, $\omega_{0}=10 \mathrm{~T}$, its magnitude at $T_{c}$ is in agreement with that obtained in ChPT for massive pion gas system in Ref. [47]. Qualitatively, the bulk viscosity corresponds to nonconformality, thus it is reasonable to observe a sharp rising of bulk viscosity near phase transition. Ref. 47] has investigated the correlation between the bulk viscosity and conformal breaking, and supports the results in Ref.42, 43]. The sharp rising of bulk viscosity has also been observed by another lattice result 48 and in the linear sigma model 496. However, till now, no full calculation has been done for the bulk viscosity. The frequency dependence of the spectral density has been analyzed in Refs. 50. and 51] and the limitation of the ansatz Eq.(29) has been discussed. From Eq. (28), we see that the bulk viscosity is dominated by $C_{v}$ at $T_{c}$. If $C_{v}$ diverges at $T_{c}$, the bulk viscosity should also be divergent at the critical point and behave as $t^{-\alpha}$. However, the detailed analysis in the Ising model in Ref. [52] shows a very different divergent behavior $\zeta \sim t^{-z \nu+\alpha}$, with $z \simeq 3$ the dynamic critical exponent and $\nu \simeq 0.630$ the critical exponent in the Ising system. More careful calculation on the bulk viscosity is needed in the future.

\section{SUMMARY AND DISCUSSION}

In this paper, we have extended the linear sigma model with three quark flavors to include certain aspects of gluon dynamics via the Polyakov loop. The PLSM model encodes two basic features that govern low energy QCD, spontaneous chiral symmetry breaking and confinement, and is a framework proposed to correctly interpret results from QCD thermodynamics and extrapolate to regions not accessible by lattice computations.

Within the mean field approximation, we have studied the $(T, \mu)$ phase diagram of the Polyakov linear sigma model with the polynomial form of the Polyakov loop potential. It is found that in the linear sigma model with Polyakov loop, the three phase transitions, i.e, the chiral restoration of $u, d$ quarks, the chiral restoration of $s$ quark, and the deconfinement phase transition are independent and happen at different critical temperatures. It is found that in the PLSM, at low density, there exists two-flavor quarkyonic phase, where the $u, d$ quarks restore chiral symmetry but still in confinement, and at high density there exists three-flavor quarkyonic phase where $u, d$ quarks restore chiral symmetry but still in confinement. The linear sigma model with and without the Polyakov loop has a general feature: there is a crossover in the low density region and turn a first-order phase transition in the high-density region accompanied by a critical end point (CEP). Here we observe large discrepancies for the critical end point between the PLSM and LSM models. For the LSM model, the critical point is found at $\left(T_{E}, \mu_{E}\right) \simeq(92.5,216) \mathrm{MeV}$, while that of the PLSM model is at $\left(T_{E}, \mu_{E}\right) \simeq(188,139.5) \mathrm{MeV}$, which is close to the lattice result $\left(T_{E}, \mu_{E}\right)=\left(162 \pm 2 \mathrm{MeV}, \mu_{E}=\right.$ $120 \pm 13 \mathrm{MeV}$ ) [40]. The critical chemical potential $\mu_{E}$ in PLSM is much lower than that in the PNJL model with three quark flavors where the predicted critical end point is at $\mu_{E}>300 \mathrm{MeV}[23,24]$.

In order to compare our results with the lattice QCD simulations and other models, at zero chemical potential but finite temperature, we have investigated the thermodynamic properties and bulk viscosity in the PLSM and LSM model. It is found that the inclusion of the Polyakov loop is necessary in order to quantitatively fit the lattice QCD result in the pure gauge sector. Our results in the PLSM and LSM models show that at critical temperature $T_{c}$, the trace anomaly $\Delta$, the specific heat $C_{v}$ show upward cusp at $T_{c}$. The ratio of pressure density over energy density $p / \varepsilon$ and the square of the sound velocity $C_{s}^{2}$ show downward cusp at $T_{c}$. These cusp behaviors at phase transition resemble lattice QCD results. We find that the PLSM model can reproduce all the thermodynamic and transport properties of the hot quark-gluon system near the critical temperature. $p / \varepsilon$ at $T_{c}$ is close to the lattice QCD results 0.075 , the trace anomaly $\Delta \simeq 6.6$ at $1.3 T_{c}$ is close to the lattice result of the peak value 7.7 , the bulk viscosity to entropy density ratio $\zeta / s$ at $T_{c}$ is around 0.2 , which is also agreement with the lattice QCD result in Ref. 443, 48.

In this work the thermodynamics of the PLSM model have been compared to three-flavor lattice data, and it has been shown that the results of the model indeed agree with the lattice data, but it should be pointed that the used quark masses are different from each other, due to the fact that the lattice data with large quark masses while our model calculations physical values have been used. Furthermore because the model has several adjustable parameters, the numerical results of the critical temperature and the critical chemical potential of the phase transitions are, of course, parameter dependent. As above discussions for small temperatures and finite chemical potentials the chiral phase transition is probably of first-order while a crossover is expected at high temperatures and small chemical potentials, this suggests the existence of at least one critical endpoint (CEP) where the first-order transition line in the phase diagram terminates. For the lattice simulations, although there are much progress have been achieved in the lattice studies for the QCD thermodynamics, at finite chemical potentials the fermion sigma problem is still a considerable obstacle, some lattice groups differ in their predictions. For the "CEP" problem, the location and even the possible existence in the phase diagram is still an open question [53] [54], in such a manner, a comparison to the lattice data in Ref. 40] is less meaningful.

It is interesting to mention some further developments for this work, such as extensions beyond mean field theory by using loop expansion[31]32] or concerning both Polyakov loop and mesonic fluctuations [55], and consideration 
about matter at high baryon density for asymmetric quark matter, and such discussions are more relevant to the real experiment (RHIC and LHC) or observation (neutron stars), all of above extensions leave much room for further detailed investigations. Especially, at high chemical potential with asymmetric quark matter $\mu_{u} \neq \mu_{d} \neq \mu_{s}$, since in

that case we can discuss the pion condensate and kaon condensate in the Polyakov linear sigma model [28, 56, 57. Eventually, work in this direction is in progress.

\section{Acknowledgments}

We thank S. He, B.C. Li, T. Kahara and Z. Zhang for valuable discussions. The work of H.M. and J.J. is supported by NSFC 10904029,10905014 and the Natural Science Foundation of Zhejiang Province under Grant No. Y7080056, Y6090345. The work of M.H. is supported by CAS program "Outstanding young scientists abroad brought-in", CAS key project KJCX3-SYW-N2, NSFC10735040, NSFC10875134, and the support of K.C.Wong Education Foundation, Hong Kong.

[1] D. H. Rischke, Prog. Part. Nucl. Phys. 52, 197 (2004).

[2] G. 't Hooft, Phys. Rev. Lett. 37, 8 (1976).

[3] G. 't Hooft, Phys. Rev. D 14, 3432 (1976) [Erratum-ibid. D 18, 2199 (1978)].

[4] D. Roder, J. Ruppert and D. H. Rischke, Phys. Rev. D 68, 016003 (2003)

[5] J. T. Lenaghan, D. H. Rischke and J. Schaffner-Bielich, Phys. Rev. D 62, 085008 (2000).

[6] F. Karsch, Lect. Notes Phys. 583, 209 (2002).

[7] A. M. Polyakov, Phys. Lett. B 72 (1978) 477.

[8] L. Susskind, Phys. Rev. D 20, 2610 (1979).

[9] B. Svetitsky and L. G. Yaffe, Nucl. Phys. B 210, 423 (1982).

[10] B. Svetitsky, Phys. Rept. 132, 1 (1986).

[11] K. Fukushima, Annals Phys. 304, 72 (2003).

[12] K. Fukushima, Phys. Lett. B 591, 277 (2004).

[13] C. Ratti, M. A. Thaler and W. Weise, Phys. Rev. D 73, 014019 (2006).

[14] K. Fukushima, Phys. Rev. D 77, 114028 (2008); [Erratum-ibid. D 78, 039902 (2008)].

[15] B. J. Schaefer, J. M. Pawlowski and J. Wambach, Phys. Rev. D 76, 074023 (2007).

[16] T. Kahara and K. Tuominen, Phys. Rev. D 78, 034015 (2008).

[17] B. J. Schaefer and M. Wagner, arXiv:0812.2855 [hep-ph].

[18] E. Megias, E. Ruiz Arriola and L. L. Salcedo, Phys. Rev. D 74, 065005 (2006).

[19] S. K. Ghosh, T. K. Mukherjee, M. G. Mustafa and R. Ray, Phys. Rev. D 73, 114007 (2006).

[20] C. Ratti, S. Roessner, M. A. Thaler and W. Weise, Eur. Phys. J. C 49, 213 (2007).

[21] S. Mukherjee, M. G. Mustafa and R. Ray, Phys. Rev. D 75, 094015 (2007).

[22] S. Roessner, C. Ratti and W. Weise, Phys. Rev. D 75, 034007 (2007).

[23] W. j. Fu, Z. Zhang and Y. x. Liu, Phys. Rev. D 77, 014006 (2008).

[24] M. Ciminale, R. Gatto, N. D. Ippolito, G. Nardulli and M. Ruggieri, Phys. Rev. D 77, 054023 (2008).

[25] H. Abuki, R. Anglani, R. Gatto, G. Nardulli and M. Ruggieri, Phys. Rev. D 78, 034034 (2008).

[26] D. Gomez Dumm, D. B. Blaschke, A. G. Grunfeld and N. N. Scoccola, Phys. Rev. D 78, 114021 (2008).

[27] W. j. Fu and Y. x. Liu, Phys. Rev. D 79, 074011 (2009).

[28] Z. Zhang and Y. X. Liu, Phys. Rev. C 75, 064910 (2007).

[29] C. Sasaki, B. Friman and K. Redlich, Phys. Rev. D 75, 074013 (2007).

[30] C. Ratti, S. Roessner and W. Weise, Phys. Lett. B 649, 57 (2007).

[31] T. Herpay and Z. Szep, Phys. Rev. D 74, 025008 (2006).

[32] J. M. Cornwall, R. Jackiw and E. Tomboulis, Phys. Rev. D 10, 2428 (1974).

[33] M. Cheng et al., Phys. Rev. D 77, 014511 (2008).

[34] B. J. Schaefer and M. Wagner, Phys. Rev. D 79, 014018 (2009).

[35] J. T. Lenaghan, Phys. Rev. D 63, 037901 (2001).

[36] O. Scavenius, A. Mocsy, I. N. Mishustin and D. H. Rischke, Phys. Rev. C 64, 045202 (2001)

[37] J. I. Kapusta and C. Gale, "Finite-temperature field theory: Principles and applications," ( Cambridge University Press, UK, 2006).

[38] K. Fukushima and Y. Hidaka, Phys. Rev. D 75, 036002 (2007) [arXiv:hep-ph/0610323].

[39] H. Abuki and K. Fukushima, arXiv:0901.4821 [hep-ph].

[40] Z. Fodor and S. D. Katz, JHEP 0404, 050 (2004) [arXiv:hep-lat/0402006].

[41] L. McLerran and R. D. Pisarski, Nucl. Phys. A 796, 83 (2007) [arXiv:0706.2191 [hep-ph]].

[42] D. Kharzeev and K. Tuchin, JHEP 0809, 093 (2008).

[43] F. Karsch, D. Kharzeev and K. Tuchin, Phys. Lett. B 663, 217 (2008). 
[44] B. C. Li and M. Huang, arXiv:0903.3650 [hep-ph], and references therein.

[45] G. Torrieri and I. Mishustin, Phys. Rev. C 78, 021901 (2008).

[46] G. Torrieri, B. Tomasik and I. Mishustin, Phys. Rev. C 77, 034903 (2008).

[47] D. Fernandez-Fraile and A. G. Nicola, Phys. Rev. Lett. 102, 121601 (2009) [arXiv:0809.4663 [hep-ph]], D. Fernandez-Fraile and A. Gomez Nicola, Eur. Phys. J. C 62, 37 (2009) [arXiv:0902.4829 [hep-ph]].

[48] H. B. Meyer, Phys. Rev. Lett. 100, 162001 (2008).

[49] K. Paech and S. Pratt, Phys. Rev. C 74, 014901 (2006).

[50] G. D. Moore and O. Saremi, JHEP 0809, 015 (2008)

[51] K. Huebner, F. Karsch and C. Pica, arXiv:0808.1127 [hep-lat].

[52] A. Onuki, Phys. Rev. E 55, 403 (1997).

[53] S. Ejiri, Phys. Rev. D 73, 054502 (2006).

[54] M. A. Stephanov, PoS LAT2006, 024 (2006).

[55] S. Roessner, T. Hell, C. Ratti and W. Weise, Nucl. Phys. A 814, 118 (2008).

[56] J. O. Andersen, Phys. Rev. D 75, 065011 (2007).

[57] T. H. Phat, N. V. Long, N. T. Anh and L. V. Hoa, Phys. Rev. D 78, 105016 (2008). 